\title{
Dynamic Simulation Analysis of a Motorcycle Suspension System - Assessment of Comfort
}

Stefan Segla, Sayantan Roy

Faculty of Mechanical Engineering, Technical University of Kosice. Letna 9, 04200 Kosice. Slovakia. E-mail: stefan.segla@tuke.sk

The paper deals with dynamic in-plane simulation analysis of a motorcycle suspension. The motorcycle's mechanical model is considered as a visco-elastically suspended rigid body. Two types of the kinematic excitation are considered - a deterministic „hat“ shaped bump and stochastically uneven road characterized by its power spectral density. The simulation results for both the deterministic bump and stochastically uneven road show that significant reduction of the root mean square value of the motorcycle body centroid acceleration (comfort criterion) can be achieved by placing the lower end point of the rare spring-damper module closer to the beginning of the swinging arm and also by increasing deviation (tilt) of the spring-damper module from the vertical. The maximum improvement in the root mean square value of the motorcycle body centroid acceleration is $51.7 \%$ for the deterministic „hat" shaped bump and $37.4 \%$ for the stochastically uneven road. The method presented in the paper can be employed in design of both touring motorcycles, which are characterized by higher requirements of comfort, and off-road motorcycles where protection from impacts generated by bumps is important.

Keywords: Motorcycle, Suspension System, Simulation, Road Profile, Comfort

\section{Introduction}

A motorcycle can be considered as a rigid body connected to the front and rear wheels with springdamper modules. The rigid motorcycle body, composed of chassis, engine, steering head and rider, constitutes the sprung mass. The wheels and masses attached to them constitute the unsprung masses. A motorcycle suspension serves the following main purposes:

- provides comfort to the rider by keeping him comfortably isolated from the vibrations generated by the interaction of the wheels with the uneven road,

- ensures wheel grip on uneven road in order to transmit the required driving, braking and also lateral forces.

The degree of required comfort depends on the use of the motorcycle. Comfort of racing motorcycles is less important than the motorcycle's capability of keeping the wheels in the sufficient contact with the road. Touring motorcycles are characterized by higher requirements of comfort. In off-road motorcycles the suspension serves to isolate the sprung mass from impacts generated by jumps. Suspensions in these motorcycles have greater wheel travel than in touring motorcycles.

A grey-box model of a motorcycle mono-tube shock absorber is proposed in [1]. It consists of a nonlinear parametric model and a black-box neuralnetwork-based model. The shock absorber model is experimentally validated. In [2] simulation and experimental investigation of motorcycle dynamic characteristics for displacement-sensitive shock absorber is presented. Dynamics behaviours of motorcycles in turns is studied in [3] and [4]. Sharma and Limbeer in [5] and [6] describe a design methodology for the suspension of a novel aerodynamically efficient motorcycle. Optimization of passive vehicle suspensions for improving the ride comfort of a motorcycle driver is investigated in [7]. The paper [8] deals with modelling, dynamic analysis and optimization of stiffness and damping parameters of the main suspension of the Škoda 21Tr trolleybus. In [9] it is shown how the kinematic excitation caused by uneven roads together with the design parameters of a vehicle suspension (stiffnesses of the suspension springs, damping coefficients and tire stiffnesses) affect the comfort of the driver and passengers, safety of the ride and relative displacements between the sprung and unsprung masses. In [10] modelling and optimization of a passive seat suspension with a vibration absorber is investigated.

\section{Mechanical and Mathematical Model}

The motorcycle's mechanical model, considered as a viscoelastically suspended rigid body [11], [12] consists of the motorcycle rigid body (sprung mass) 1 , the 
front (2) and rare (3) wheels (unsprung masses) and the front and rare spring-damper modules $(4,5)$ between the sprung and unsprung masses, Fig. 1.

The motorcycle motion can be described by four independent coordinates $z_{1}, z_{2}, z_{3}, \varphi_{3}$, Fig. 2, where $z_{1}$ and $z_{2}$ are the vertical displacements of the front and rare unsprung masses (the equivalent masses at the center of the wheels), $z_{3}$ is the vertical displacement of the sprung mass centroid $\mathrm{T}_{3}$ and $\varphi_{3}$ defines the pitching angular displacement of the sprung mass.

The equations of motion of the motorcycle can be described by the following set of ordinary differential equations

$$
\begin{gathered}
m_{1} \ddot{1}_{1}-b_{1, e q}\left(\dot{z}_{3}+l_{1} \dot{\varphi}_{3}-\dot{z}_{1}\right)-k_{1, e q}\left(z_{3}+l_{1} \varphi_{3}-z_{1}\right)+k_{3}\left(z_{1}-u_{1}\right)=0, \\
m_{2} \ddot{z}_{2}-b_{2, e q}\left(\dot{z}_{3}-l_{2} \dot{\varphi}_{3}-\dot{z}_{2}\right)-k_{2, e q}\left(z_{3}-l_{2} \varphi_{3}-z_{2}\right)+k_{4}\left(z_{2}-u_{2}\right)=0, \\
m_{3} \ddot{z}_{3}+b_{1, e q}\left(\dot{z}_{3}+l_{1} \dot{\varphi}_{3}-\dot{z}_{1}\right)+b_{2, e q}\left(\dot{z}_{3}-l_{2} \dot{\varphi}_{3}-\dot{z}_{2}\right)+k_{1, e q}\left(z_{3}+l_{1} \varphi_{3}-z_{1}\right)+k_{2, e q}\left(z_{3}-l_{2} \varphi_{3}-z_{2}\right)=0, \\
I_{3} \ddot{\varphi}_{3}+b_{1, e q} l_{1}\left(\dot{z}_{3}+l_{1} \dot{\varphi}_{3}-\dot{z}_{1}\right)-b_{2, e q} l_{2}\left(\dot{z}_{3}-l_{2} \dot{\varphi}_{3}-\dot{z}_{2}\right)+k_{1, e q} l_{1}\left(z_{3}+l_{1} \varphi_{3}-z_{1}\right)-k_{2, e q} l_{2}\left(z_{3}-l_{2} \varphi_{3}-z_{2}\right)=0,
\end{gathered}
$$

where the coordinates $u_{1}$ and $u_{2}$ describe the uneven road profile (kinematic excitation), Fig. 2.

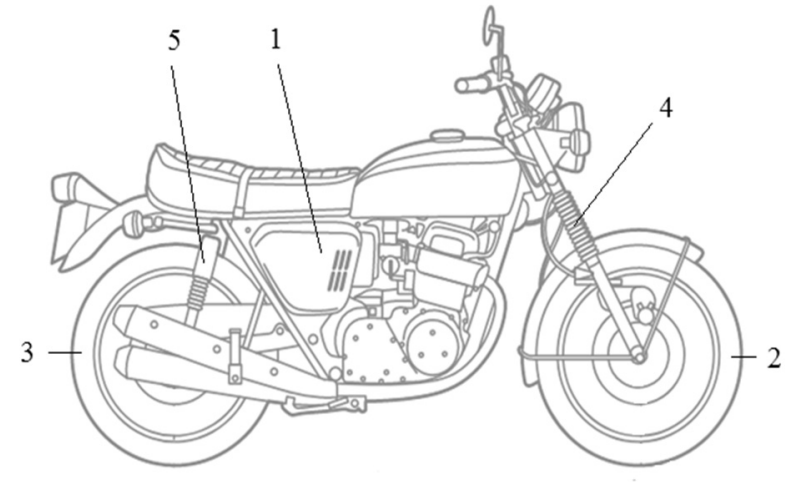

Fig. 1 Motorycle Components

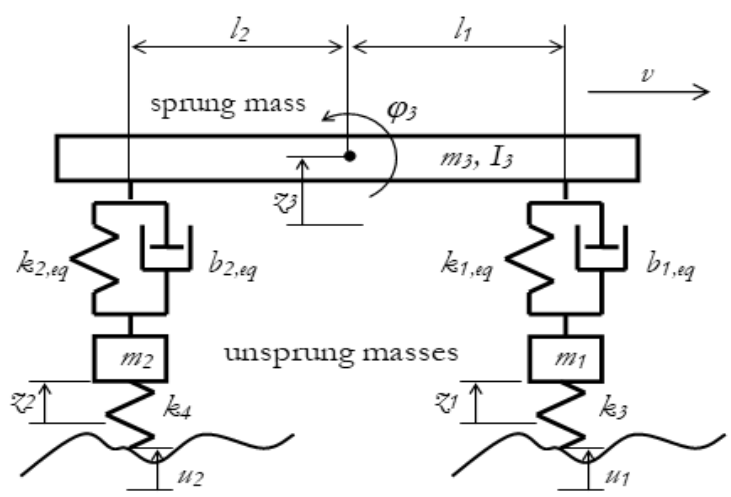

Fig. 2 Mechanical Model of the Motorycle

\subsection{Equivalent Stiffness and Damping Coefficients - Front Suspension}

The potential energy of the original tilted spring $k_{1}$ , Fig. 3, can be expressed by the approximate equation

$$
V_{1, o r}=\frac{1}{2} k_{1}\left[\left(z_{3}+l_{1} \varphi_{3}\right) \cos \varepsilon_{1}-z_{1} \cos \varepsilon_{1}\right]^{2}=\frac{1}{2} k_{1}\left(z_{3}+l_{1} \varphi_{3}-z_{1}\right)^{2} \cos ^{2} \varepsilon_{1}
$$

and the potential energy of the equivalent spring $k_{1, e q}$ is

$$
V_{1, e q}=\frac{1}{2} k_{1, e q}\left(z_{3}+l_{1} \varphi_{3}-z_{1}\right)^{2} .
$$

Equality of the potential energy $V_{1, o r}$ and $V_{1, e q}$ leads to the dependence between the stiffness coefficient $k_{1, e q}$ of the equivalent spring and the stiffness coefficient of the original spring $k_{1}$

$$
k_{1, e q}=k_{1} \cos ^{2} \varepsilon_{1}
$$

Using the dissipative function it is possible to derive analogical equation for the damping coefficient

$$
b_{1, e q}=b_{1} \cos ^{2} \varepsilon_{1} .
$$

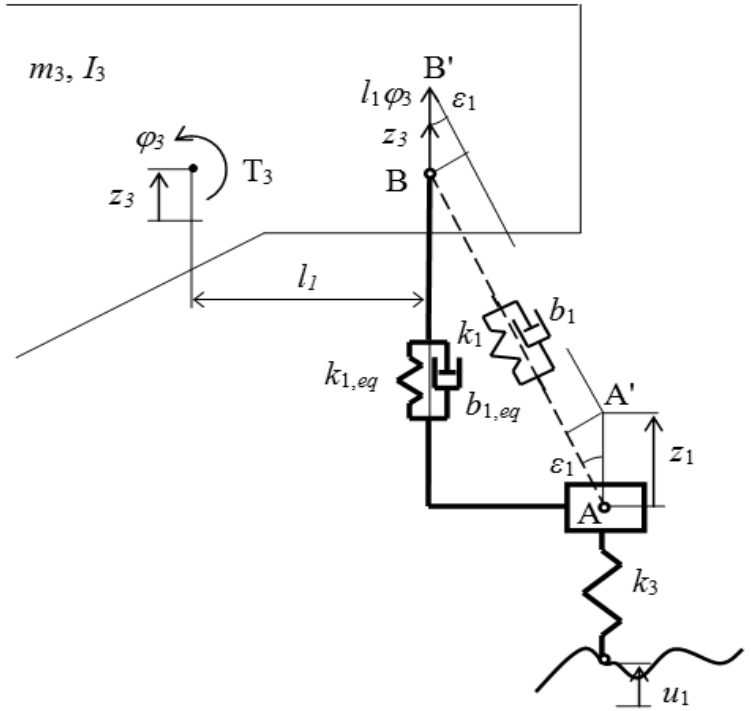

Fig. 3 Determining the Equivalent Stiffness and Damping Coefficients - the Front Suspension 
2.2 Equivalent Stiffness and Damping Coefficients Rare Ssuspension

The potential energy of the original tilted spring

$$
V_{2, o r}=\frac{1}{2} k_{2}\left[\left(z_{3}-\bar{l}_{2} \varphi_{3}\right) \cos \varepsilon_{2}-x \cos \varepsilon_{2}\right]^{2}=\frac{1}{2} k_{2}\left(z_{3}-\bar{l}_{2} \varphi_{3}-x\right)^{2} \cos ^{2} \varepsilon_{2}
$$

The potential energy of the vertical spring $\bar{k}_{2, e q}$ is

$$
\bar{V}_{2, e q}=\frac{1}{2} \bar{k}_{2, e q}\left(z_{3}-\bar{l}_{2} \varphi_{3}-x\right)^{2}
$$

Equality of $V_{2, o r}$ and $\bar{V}_{2, e q}$ gives

$$
\bar{k}_{2, e q}=k_{2} \cos ^{2} \varepsilon_{2} \text {. }
$$

Using dissipative function we get

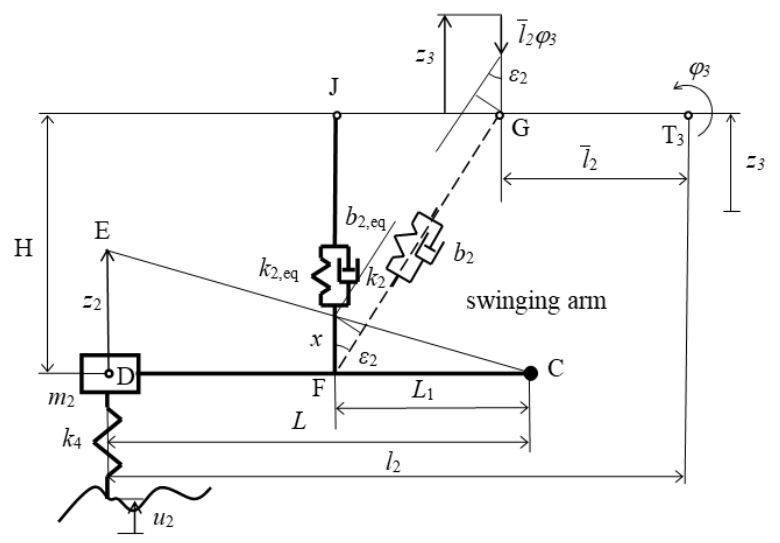

Fig. 4 Determining the Equivalent Stiffness and Damping Coefficients of the Rare Suspension, part $A$

$$
\bar{b}_{2, e q}=b_{2} \cos ^{2} \varepsilon_{2} \text {. }
$$

Now the vertical spring-damper module $\left(\bar{k}_{2, e q}\right.$, $\bar{b}_{2, e q}$ ) will be replaced by the spring-damper module ( $\left.k_{2, e q}, b_{2, e q}\right)$ acting on the mass $m_{2}$, Fig. 5.

For the potential energy in the spring $\bar{k}_{2, e q}$ we can write, Fig. 5

$$
\tilde{V}_{2, o r}=\frac{1}{2} \bar{k}_{2, e q} x^{2}
$$

where

$$
x=\frac{L_{1}}{L} z_{2} .
$$

Substituting equation (14) into equation (13) we get

$$
\tilde{V}_{2, o r}=\frac{1}{2} \bar{k}_{2, e q} z_{2}^{2}\left(\frac{L_{1}}{L}\right)^{2}
$$

$k_{2}$, Fig. 4 , can be expressed by the approximate equation

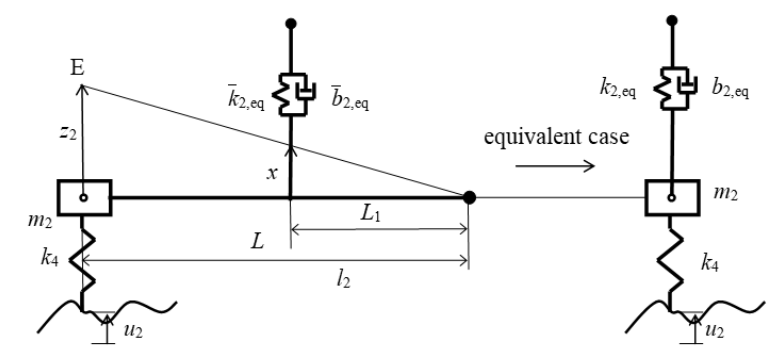

Fig. 5 Determining the Equivalent Stiffness and Damping Coefficients of the Rare Suspension, part B

For the potential energy of the spring $k_{2, e q}$ of the equivalent case, Fig. 5 , the following equation holds

$$
\tilde{V}_{2, e q}=\frac{1}{2} k_{2, e q} z_{2}^{2}
$$

Equality of equations (16) and (15) gives

$$
k_{2, e q}=\bar{k}_{2, e q}\left(\frac{L_{1}}{L}\right)^{2}
$$

Substituting $\bar{k}_{2, e q}$, equation (11), into equation (17) gives

$$
k_{2, e q}=k_{2}\left(\frac{L_{1}}{L}\right)^{2} \cos ^{2} \varepsilon_{2}
$$

Using dissipative function we get

$$
b_{2, e q}=b_{2}\left(\frac{L_{1}}{L}\right)^{2} \cos ^{2} \varepsilon_{2}
$$

\section{Deterministic and Stochastic Road Profi- les}

The most important source of excitation is caused by the road unevenness. It can be divided into two basic types - deterministic and stochastic ones, as described e.g. in [13].

A suitable deterministic non-normalized road unevenness is of the so-called „hat" shape, which to some extent respect the actual shape of the tyre. It suits to the point road-tyre contact modelling. Mathematical model of the bump, depending on the longitudinal coordinate $x$, is described by equation 


$$
u(x)=\frac{h_{m}}{2}\left(1-\cos \frac{2 \pi}{d} x\right) \text { for } 0 \leq x \leq d, \text { otherwise } u(x)=0
$$

where is: $R$ - radius of the circumscribed circle, Fig. $6, d$ - the length of the bump, $h_{m}$ - the height of

$$
u(t)=\frac{h_{m}}{2}\left(1-\cos \frac{2 \pi}{T} t\right) \text { for } 0 \leq t \leq T \text {, otherwise } u(t)=0,
$$

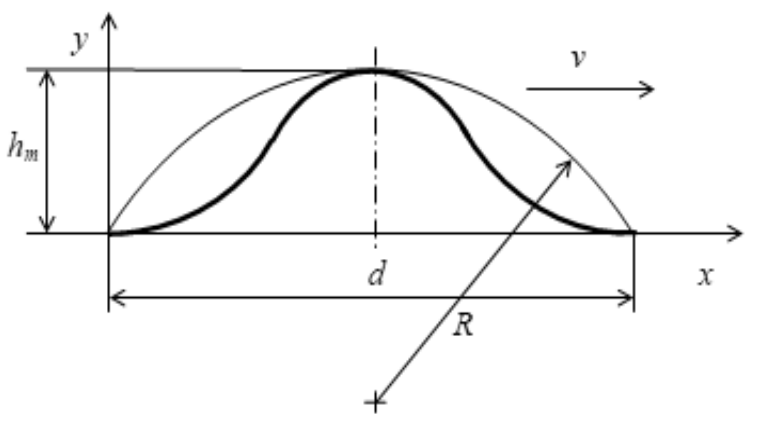

Fig. 6 Deterministic ,hat" shaped bump

where $T=d / v, t=x / v, v$ is the motorcycle speed and $T$ is the bump transit time.

Modelling of the stochastic road is based on the idea that each stochastic stationary process can be represented by the sum of cosine functions. The theory of the method [13] was developed in 1971 by Shinozuka.

\section{Results of Simulations}

Parameters of the motorcycle mechanical model are: $m_{1}=15 \mathrm{~kg}, m_{2}=18 \mathrm{~kg}, m_{3}=194 \mathrm{~kg}$,

$I_{3}=38 \mathrm{~kg} \cdot \mathrm{m}^{2}, k_{1}=15000 \mathrm{~N} / \mathrm{m}$, $k_{2}=24000 \mathrm{~N} / \mathrm{m}$, $k_{3}=180000 \mathrm{~N} / \mathrm{m}$, $k_{4}=180000 \mathrm{~N} / \mathrm{m}$,

$b_{1}=710 \mathrm{~N} \cdot \mathrm{s} / \mathrm{m}$, $b_{2}=1171 \mathrm{~N} \cdot \mathrm{s} / \mathrm{m}$, $l_{1}=0.64 \mathrm{~m}$,

$l_{2}=0.7 \mathrm{~m}, \varepsilon_{1}=27^{\circ}, H=0.6 \mathrm{~m}, L=0.6 \mathrm{~m}$. For the „hat" shaped bump the following prameters were used: $d=2.68 \mathrm{~m}, h_{m}=0.06 \mathrm{~m}, v=11 \mathrm{~m} / \mathrm{s}$. When driving on a stochastically uneven road the parameters as follows were used: $S_{u}\left(\Omega_{0}\right)=22.10^{-6} \mathrm{~m}^{3}$ (power spectral density of an asphalt-concrete road of average quality), $v=20 \mathrm{~m} / \mathrm{s}$.

In Tab. 1 root mean square (rms) values of the motorcycle body centroid acceleration and displacement and maximum displacement amplitude for combinations of different values of 2 parameters $\varepsilon_{2}$ and $L_{1}$ for the ride over the ,hat" shaped bump are shown.

Tab. 2 shows the rms values of the motorcycle body centroid acceleration and displacement for combinations of different values of the same parameters $\varepsilon_{2}$ and $L_{1}$ as in the previous case, for the ride along a stochastically uneven road.
Tab. 1 The rms V alues of the Motorcycle Body Centroid Acceleration, Displacement and Maximum Displacement Amplitude - the Ride Over the „Hat" Shaped Bump

\begin{tabular}{|c|c|c|c|c|}
\hline$\varepsilon_{2}\left[{ }^{\circ}\right]$ & $\begin{array}{c}L_{1} \\
{[\mathrm{~m}]}\end{array}$ & $\begin{array}{c}\ddot{z}_{3, \text { ef }} \\
{\left[\mathrm{m} / \mathrm{s}^{2}\right]}\end{array}$ & $\begin{array}{c}z_{3, \text { ef }} \\
{[\mathrm{m}]}\end{array}$ & $\begin{array}{c}z_{3, \mathrm{max}} \\
{[\mathrm{m}]}\end{array}$ \\
\hline 0 & 0.2 & 0.02615 & 0.00036 & 0.025 \\
\hline 20 & 0.2 & 0.02646 & 0.00035 & 0.025 \\
\hline 40 & 0.2 & 0.02767 & 0.00035 & 0.023 \\
\hline 0 & 0.4 & 0.03754 & 0.00039 & 0.040 \\
\hline 20 & 0.4 & 0.03504 & 0.00039 & 0.039 \\
\hline 40 & 0.4 & 0.02856 & 0.00038 & 0.038 \\
\hline 0 & 0.6 & 0.05412 & 0.00039 & 0.048 \\
\hline 20 & 0.6 & 0.05176 & 0.00039 & 0.047 \\
\hline 40 & 0.6 & 0.04340 & 0.00039 & 0.043 \\
\hline
\end{tabular}

Tab. 2 The rms V alues of the Motorcycle Body Centroid Acceleration and Displacement - the Ride Along a Stochastically Uneven Road

\begin{tabular}{|c|c|c|c|}
\hline$\varepsilon_{2}\left[{ }^{\circ}\right]$ & $L_{1}[\mathrm{~m}]$ & $\ddot{z}_{3, r m s}\left[\mathrm{~m} / \mathrm{s}^{2}\right]$ & $z_{3, r m s}[\mathrm{~m}]$ \\
\hline 0 & 0.2 & 0.04625 & 0.00059 \\
\hline 20 & 0.2 & 0.04598 & 0.00060 \\
\hline 40 & 0.2 & 0.04511 & 0.00063 \\
\hline 0 & 0.4 & 0.05631 & 0.00056 \\
\hline 20 & 0.4 & 0.05459 & 0.00056 \\
\hline 40 & 0.4 & 0.05022 & 0.00057 \\
\hline 0 & 0.6 & 0.07208 & 0.00055 \\
\hline 20 & 0.6 & 0.06918 & 0.00055 \\
\hline 40 & 0.6 & 0.06084 & 0.00056 \\
\hline
\end{tabular}

Graph 1 shows the dependence of the centroid $T_{3}$ vertical displacement $z_{3}$ on time for the ride over the „hat" shaped bump for two different cases described in the graph.

The maximum improvement in the rms value of the motorcycle body centroid acceleration is $51.7 \%$ for the deterministic ,hat" shaped bump and 37.4\% for the stochastically uneven road. The rms value of the body centroid displacement remains practically constant.

\section{Conclusions}

The results presented in the paper show that in both ride over a ,hat" shaped bump and along a stochastically uneven road significant reduction of the rms value of the motorcycle body centroid can be achieved by placing the lower end point of the spring- 
damper module closer to the beginning of the swinging arm. Significant reduction of the value can be also achieved by increasing deviation (tilt) of the springdamper module from the vertical, but this reduction is decreasing with placing the lower end point of the rare spring-damper module closer to the beginning of the swinging arm. The rms value of the body centroid displacement remains practically constant. The above conclusions are important for improving comfort of the motorcycle rider, because the centroid acceleration is a decisive factor determining the motorcycle comfort level.

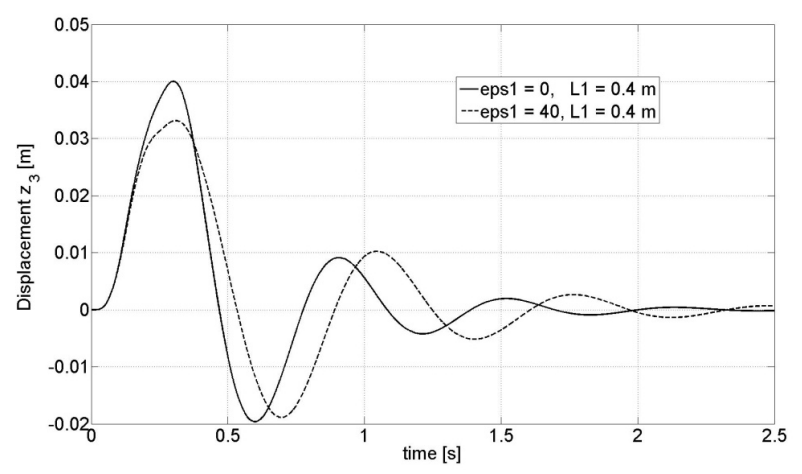

Graph 1 Dependence of $z_{3}$ on Time - „Hat" Shaped Bump

\section{Acknowledgement}

The authors were supported by grant project VEGA No. 1/0290/18.

\section{References}

[1] BEGHI, A., LIBERATI, M., MEZZALIRA, S., PERON, S. (2007). Grey-box modelling of a motorcycle shock absorber for virtual prototyping applications. In: Simulation Modeling Practice and Theory, Vol. 15, pp. 894-907. Elsevier B.V. ISSN: 1569-190X.

[2] LEE, Ch.T., MOON, B.Y. (2006). Simulation and experimental validation of vehicle dynamic characteristics for displacement-sensitive shock absorber using fluid-flow modelling. In: Mechanical Systems and Signal Processing, Vol. 20, pp. 373-388. Elsevier Ltd. ISSN: 0888-3270.

[3] SHARP, R.S. (2012). Rider control of a motorcycle near to its cornering limits. In: Vebicle System Dynamics, Vol. 50, No. 8, pp. 1193-1208. Taylor \& Francis. ISSN: 0042-3114.
[4] KARANAM, V.M., CHATTERJEE, A. (2011). Common underlying steering curves for motorcycles in steady turns. In: Vehicle System Dynamics, Vol. 49, No. 6, pp. 931-948. Taylor \& Francis. ISSN: 0042-3114.

[5] Optimization Toolbox For Use With MATLAB. Users Guide (2000), The MathWorks, Inc.

[6] SHAMMA, A., LIMEBEER, D.J.N. (2012). Motorcycle design using matrix inequalities and passivity constraints. In: Vebicle System Dynamics, Vol. 50, No. 3, pp. 377-393. Taylor \& Francis. ISSN: 0042-3114.

[7] SEGLA, S. (2004). Optimization of passive vehicle suspension systems for improving the ride comfort. In: Acta Mechanica Slovaca, Vol. 8, No. 3C, pp. 161-170. Faculty of Mechanical Engineering - Technical University of Košice. ISSN: 1335-2393.

[8] SEGLA, S., KAMPO, J., SOUKUP, J. (2019). Dynamic analysis and optimization of the planar model of the trolleybus Škoda 21Tr. In: Manufacturing Technology, Vol. 19, No. 3, pp. 487-491. J. E. Purkyne University in Ústí nad Labem. ISSN: 12132489.

[9] SEGLA, S., KAMPO, J. (2018). The role of modelling of road unevennesses in vehicle dynamics. In: Manufacturing Technology, Vol. 18, No. 1, pp. 124-129. J. E. Purkyne University in Ústí nad Labem. ISSN: 12132489.

[10] SEGLA, S., OREČNÝ, M., TRISOVIC, N. (2013). The role of modelling of road unevennesses in vehicle dynamics. In: Manufacturing Technology, Vol. 13, No. 4, pp. 530-534. J. E. Purkyne University in Ústí nad Labem. ISSN: 12132489.

[11] JAZAR, R.N. (2008). Vebicle Dynamics: Theory and Application, p. 1020. Springer, Melbourne. ISBN: 978-0-387-74243-4.

[12] COSSALTER, V. (2002). Motorycle Dynamics. p. 421. Vittore Cossalter, Padova.

[13] NIGAM, N.C., NARAYANAN, S. (1994). Applications of Random Vibrations, $p$. 557. Springer, New York. ISBN: 978-3540198611. 